\section{Cureus}

\title{
Association Between Smoking and Back Pain in a Cross-Section of Adult Americans
}

\author{
Bart N. Green ${ }^{1}$, Claire D. Johnson ${ }^{1}$, Jeff Snodgrass ${ }^{2}$, Monica Smith ${ }^{3}$, Andrew S. Dunn ${ }^{4}$ \\ 1. Publications, National University of Health Sciences 2. Department of Occupational Therapy, Milligan \\ College 3. Research, Life Chiropractic College West 4. Chiropractic, VA Western New York Health Care \\ System
}

$\square$ Corresponding author: Bart N. Green, bngreen@aol.com

Disclosures can be found in Additional Information at the end of the article

\section{Abstract}

Purpose: Back pain is the leading cause of global years lived with disability. This cross-sectional study assessed if a greater exposure to smoking cigarettes was associated with a greater prevalence of back pain.

Methods: This study examined data from 34,525 United States adults from the 2012 National Health Interview Survey. Analyses assessed the difference in back pain prevalence among current smokers, former smokers, and never smokers and the number of cigarettes smoked between current smokers with and without back pain.

Results: Back pain prevalence was $28 \%$. There was a significant association between back pain and smoking, $X^{2}(2,599, \mathrm{n}=34,241)=546.3, p<.001$. Back pain increased with increased smoking exposure; back pain was present in $23.5 \%$ of never-smokers, $33.1 \%$ of former smokers, and $36.9 \%$ of current smokers. The number of cigarettes smoked per day for current daily smokers was higher for those with back pain $(M d=13)$ than those without back pain $(M d=10), U$ $=2701065, z=-3.70, p<.001, r=.05$.

Conclusions: Our findings suggest that there may be a biological gradient associated with exposure to smoking cigarettes and back pain in adult Americans.

Categories: Epidemiology/Public Health

Keywords: smoking, back pain, prevalence

\section{Introduction}

Received 09/02/2016

Review began 09/12/2016 Review ended 09/16/2016 Published 09/26/2016

๑) Copyright 2016

Green et al. This is an open access article distributed under the terms of the Creative Commons Attribution License CC-BY 3.0., which permits unrestricted use, distribution, and reproduction in any medium, provided the original author and source are credited.
Back pain is highly prevalent and the leading cause of global years lived with disability [1]. Back pain is the most common chronic painful condition in Americans [2] and is associated with reduced quality of life, human suffering, and enormous financial and social burdens [3]. Approximately $\$ 34$ billion in direct cost each year is attributed to treating back pain in the United States (US) [4]. Various factors have been investigated to reduce the prevalence of back pain, including physical activity, work environment, and injury prevention strategies. It has been suggested that smoking may be associated with back pain in theoretical models [5-9], basic science studies [10-12], and epidemiological research [7, 13-14].

Inconsistent measures of association are reported in the epidemiologic studies to date and investigators have either looked at different samples or data are reported from other countries $[7,13]$. Previous reports have shown that smokers had a higher prevalence of back pain 
than former smokers and non-smokers, but the prevalence data varied by country [7], a small increased risk for back pain in smokers [15], and an increased risk of back pain in US adult smokers [14]. However, assessing the potential of a biological gradient between smoking and back pain has not been reported in US adults using data from the National Health Interview Survey (NHIS), a nationally representative cross-sectional epidemiologic survey.

Evaluation for the potential of an association between increasing levels of smoking frequency (dose) and the prevalence of back pain in US adults may help inform a better understanding of the possible interaction between smoking and back pain and to assist in designing populationbased multivariable studies to assess for risk factors for back pain. Therefore, the purpose of this study was to identify if a greater exposure to smoking cigarettes was associated with a greater prevalence of back pain in a large sample representative of the current US adult population. The study hypothesis was that the prevalence of back pain would be greater in current smokers than in former smokers and never smokers. A secondary hypothesis was that current smokers who smoked more cigarettes would report a higher prevalence of back pain than those who smoked fewer cigarettes.

\section{Materials And Methods}

The Strengthening the Reporting of Observational Studies in Epidemiology (STROBE) statement [16], as it applies to cross-sectional studies, was used to prepare this report.

\section{Study design and data source}

We performed a secondary cross-sectional analysis of US adults using data from the 2012 NHIS [17].

\section{Setting}

NHIS is an annual interview that is done in-person between trained computer-assisted interviewers and the survey respondent and the methods are reported thoroughly elsewhere [17].

\section{Participants and variables}

The population for this sample was adult (age greater than or equal to 18 years) Americans and was estimated to be approximately 313,914,000 [18]. The Sample Adult questionnaire from the 2012 NHIS was used for this study. In this questionnaire, one adult from each family interviewed was randomly selected as the 'sample adult'. Any adult was eligible to participate, except for those who were institutionalized or actively serving in the US military or unless he or she is physically or mentally unable to do so [19]. This study used publicly available and de-identified NHIS data. The research protocol was reviewed and approved prior to the commencement of data analysis by the Walden University Institutional Review Board, approval \#11-04-140058716. Participants provided consent to participate to the interviewer for the original data collection conducted by the NHIS. This data is released to the public for future secondary analyses and requires no further participant consent.

Respondents were asked if they had back pain for an entire day or longer in the previous three months. Cases were defined as those who answered "yes". Those who reported smoking at least 100 cigarettes in their lifetime were considered to be current smokers or former smokers and those who did not were considered to be never smokers. Differentiation between current smokers and former smokers was determined by the answering the question, "Do you NOW smoke cigarettes every day, some days, or not at all?” Responses were recoded into four categories of a current smoker (smoke daily or some days), former smoker, and never smoker. The number of cigarettes smoked per day was a continuous variable with a range from 0 to 99+. 


\section{Bias}

The risk of bias is present in every study and has been described as the degree to which a research report has answered the research question free from bias [20]. Hoy and colleagues identified 11 criteria to assess the risk of bias in prevalence studies, including items such as appropriate sampling, acceptable definitions of cases, rigorous data collection methods, and other items pertinent to internal and external validity [20]. Several attempts were made to minimize bias for this study. The sample size was representative of the US national population, as NHIS methodology employed a complex, randomized, and multistage sampling strategy leading to a high response rate and large sample, allowing for generalizability to the population. Typically, data for NHIS sample adults were collected directly from the subjects, which decreased the chance for bias. Cases were defined as those who answered that they had experienced back pain in the previous three months for an entire day or longer. All NHIS interviewers were trained in a standardized manner and the interview was conducted with computer assistance to decrease variation in how questions were asked.

\section{Sample size}

Using $G^{*}$ Power 3.1.9.2 for Windows (Universität Düsseldorf, Germany) [21], a power analysis was performed [22]. A medium effect size of 0.3 was selected, with alpha at 0.05 (2-tailed) and power of 0.95 , based upon the methods of Faul and colleagues [23]. The estimated number of subjects required for the Chi-square and Mann-Whitney U were 146 and 220, respectively.

\section{Statistical analysis}

All assumptions necessary to use the selected tests were met. Chi-square test for independence with weighting for population estimates was used to test the association between smoking and back pain. The Mann-Whitney U test was used to assess for a difference in the median number of cigarettes smoked per day between current daily smokers with and without back pain, current some-day smokers with and without back pain, and all current smokers (daily and some days) with and without back pain. Cases with missing data were excluded from the analysis. We used SPSS Complex Samples Analysis, version 21 (IBM Inc, Armonk, NY) for statistical testing.

\section{Results}

\section{Participants}

There were 34,525 total respondents (79.7\% response) and 284 cases $(0.8 \%)$ had missing data and were excluded from the statistical analysis, yielding a final sample of 34,241.

\section{Descriptive data}

Back pain was present in 10,078 cases (28\%). Most people had never smoked, with 6,436 current smokers in the sample (prevalence $=18 \%$ ). Descriptive statistics are presented in Table 1 . 


\section{Cureus}

\begin{tabular}{|c|c|c|c|c|c|c|}
\hline Variable & $\begin{array}{l}\text { Unweighted } \\
\text { Count }\end{array}$ & $\%$ & $\begin{array}{l}\text { Population } \\
\text { Estimate }\end{array}$ & $\begin{array}{l}\text { Standard } \\
\text { Error }\end{array}$ & $\begin{array}{l}\text { 95\% Cl Lower } \\
\text { Bound }\end{array}$ & $\begin{array}{l}\text { 95\% CI Upper } \\
\text { Bound }\end{array}$ \\
\hline \multicolumn{7}{|l|}{ Back Pain } \\
\hline Yes & 10,078 & 28.0 & $65,823,057$ & 965,737 & $63,922,579$ & 67,723,534 \\
\hline No & 24,427 & 72.0 & 168,937,180 & $1,844,349$ & $165,307,679$ & $172,566,680$ \\
\hline Total & 34,505 & 100.0 & $234,760,237$ & 2,291,291 & $230,251,198$ & $239,269,275$ \\
\hline \multicolumn{7}{|l|}{ Smoking } \\
\hline Current & 6,436 & 18.1 & $42,098,139$ & 813,046 & 40,498,141 & 43,698,136 \\
\hline Former & 7,584 & 22.2 & $51,621,850$ & 865,475 & $49,918,678$ & $53,325,021$ \\
\hline Never & 20,236 & 59.8 & $139,327,445$ & $1,617,264$ & 136,144,825 & $142,510,064$ \\
\hline Total & 34,256 & 100.0 & $233,047,434$ & 2,287,231 & 228,546,384 & $237,548,483$ \\
\hline \multicolumn{7}{|l|}{ Sex } \\
\hline Male & 15,273 & 44.2 & 113,070,897 & $1,471,455$ & $110,175,215$ & $115,966,578$ \\
\hline Female & 19,252 & 55.8 & $121,849,773$ & $1,395,324$ & $119,103,909$ & $124,595,636$ \\
\hline Total & 34,525 & 100.0 & $234,920,670$ & 2,292,625 & $230,409,005$ & 239,432,334 \\
\hline \multicolumn{7}{|l|}{ Race } \\
\hline Hispanic & 5,859 & 14.9 & $34,946,432$ & 816,213 & $33,340,203$ & $36,552,660$ \\
\hline $\begin{array}{l}\text { Non-Hispanic } \\
\text { White }\end{array}$ & 20,842 & 67.2 & $157,787,860$ & $2,071,449$ & $153,711,448$ & $161,864,271$ \\
\hline $\begin{array}{l}\text { Non-Hispanic } \\
\text { Black }\end{array}$ & 5,282 & 11.9 & 27,885,414 & 626,598 & $26,652,329$ & $29,118,498$ \\
\hline $\begin{array}{l}\text { Non-Hispanic } \\
\text { Asian }\end{array}$ & 2,168 & 5.3 & $12,415,030$ & 424,711 & $11,579,238$ & 13,250,821 \\
\hline $\begin{array}{l}\text { Non-Hispanic All } \\
\text { Other }\end{array}$ & 372 & 0.8 & $1,885,934$ & 198,313 & $1,495,671$ & $2,276,196$ \\
\hline Total & 34,525 & 100.0 & $234,920,670$ & 2,292,625 & $230,409,005$ & 239,432,334 \\
\hline
\end{tabular}

\section{TABLE 1: Descriptive Statistics for Sample and Population Estimates}

$95 \% \mathrm{Cl}=95 \%$ confidence interval

\section{Main results}

A significant association was found between back pain and smoking status, $X^{2}(2,599, \mathrm{n}=34$, 


\section{Cureus}

$241)=546.3, p<.001$. As shown in Figure 1, back pain was less prevalent in never smokers than former smokers and both of these groups had less back pain prevalence than current smokers. For current daily smokers, the Mann-Whitney $U$ test revealed a significant difference in the number of cigarettes smoked per day of those with back $(n=1,915)$ pain and those without back pain $(\mathrm{n}=3005), U=2,701,065, z=-3.70, p<.001, r=.05$ (Figure 2). For current some-day smokers, there was no difference in the number of cigarettes smoked per day of those with back pain ( $M d=3, n=485)$ and those without back pain ( $M d=3, n=919)$. When combining all smokers (daily and some-day smokers), there was no difference in the number of cigarettes smoked per day of those with back pain $(\mathrm{Md}=10, \mathrm{n}=2400)$ and those without back pain $(\mathrm{Md}=$ $10, \mathrm{n}=3924)$.

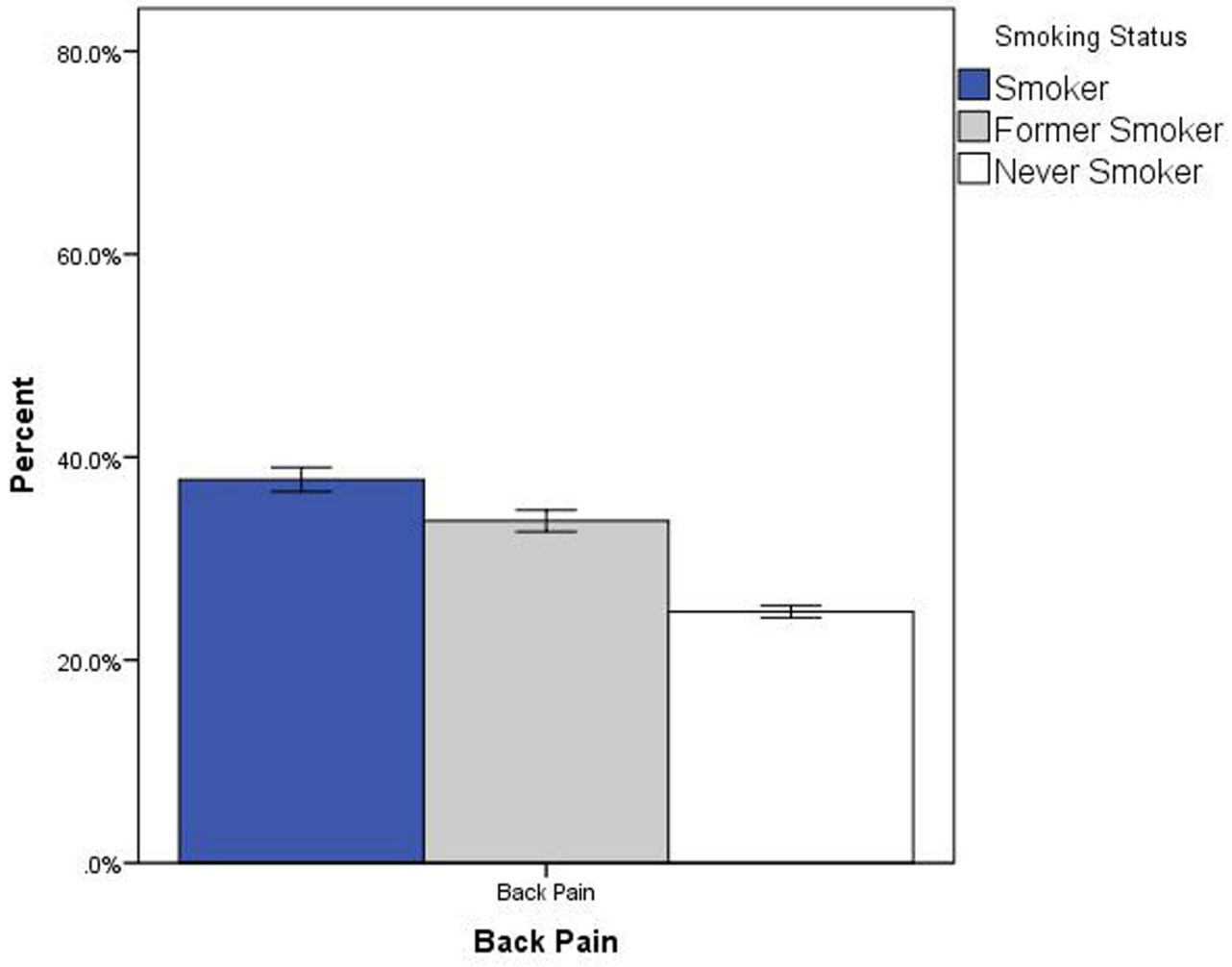

Error bars: $95 \% \mathrm{Cl}$

\section{FIGURE 1: Prevalence of Back Pain by Smoking Status}

Back pain was estimated to be present in $23.5 \%$ of never-smokers (95\% CI, $22.7-24.3$ ), $33.1 \%$ of former smokers $(95 \% \mathrm{Cl}, 31.8-34.4)$, and $36.9 \%$ of current smokers $(95 \% \mathrm{Cl}, 35.3-38.4)$ 


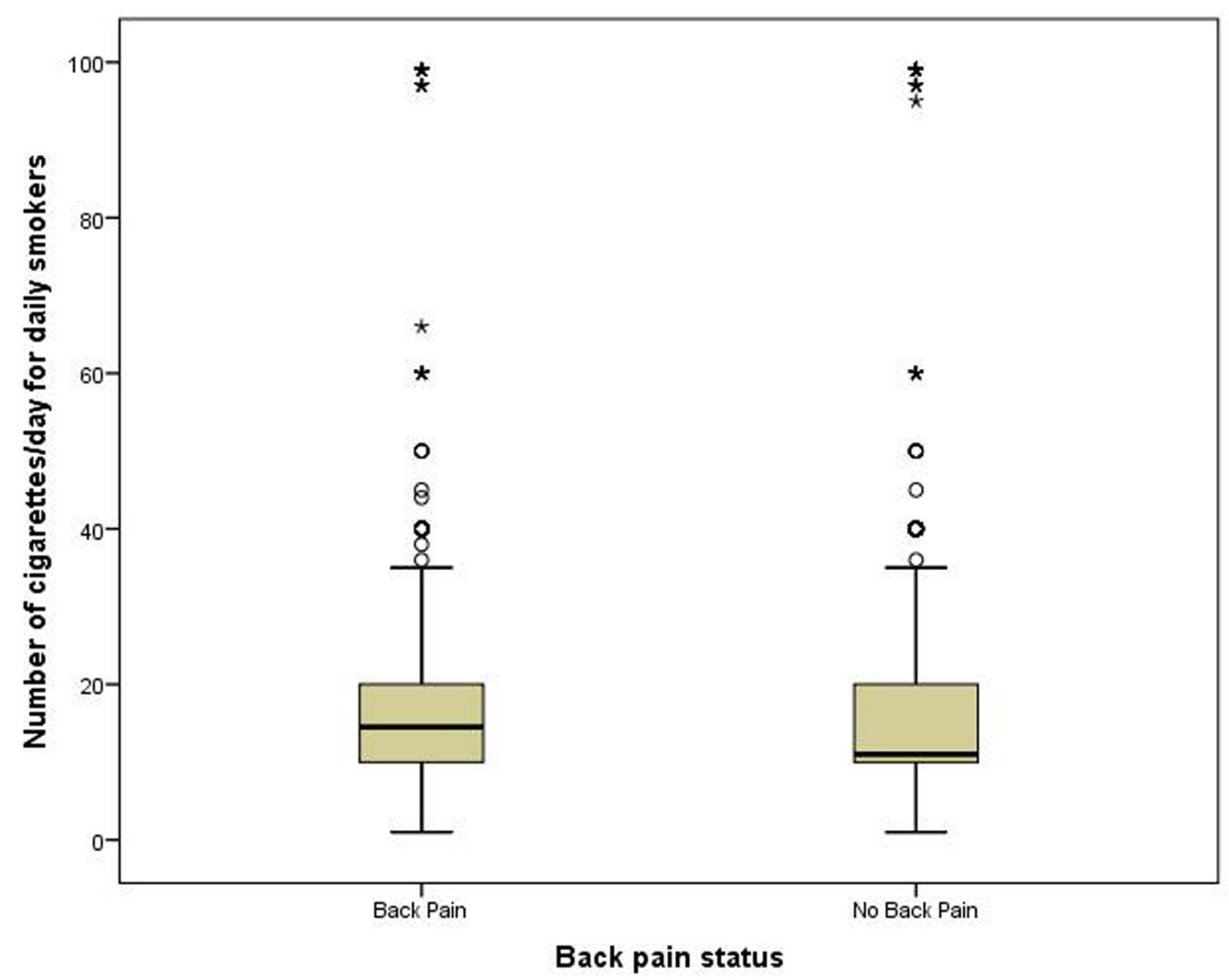

FIGURE 2: Box Plot of Median Number of Cigarettes Smoked for Daily Smokers with and without Back Pain

The median number of cigarettes smoked daily for people without back pain was 10 and for those with back pain it was 13 .

\section{Discussion}

\section{Key results}

This study found that current smokers had a higher prevalence of back pain than former smokers and never smokers and that former smokers had a higher prevalence of back pain than never smokers. Among current daily smokers, those who smoked more cigarettes per day had a statistically higher prevalence of back pain. This suggests that more frequent exposure to smoking cigarettes may possibly be associated with a higher prevalence of back pain.

\section{Interpretation}

Our findings support and add to the knowledge generated by previous US studies. One metaanalysis suggested that smokers had a higher prevalence of back pain than former smokers and non-smokers and that former smokers had a higher prevalence of back pain than non-smokers and that prevalence data varied by country and that measures of association were inconsistent across geographic regions [7]. A study of 1976-1980 data from the National Health and Nutrition Examination Survey II (NHANES II) showed a small increased risk for back pain in smokers compared to non-smokers $(\mathrm{RR}=1.13)$ with a higher prevalence of back pain among the heaviest smokers (three or more packs per day) when compared to non-smokers [15]. In this study by Deyo and Bass, the prevalence of low back pain was $10.9 \%$ versus $9.6 \%$ among those who never smoked. In their study, the one-year prevalence of back pain increased as the greatest amount 
of smoking was considered, showing a $9.6 \%$ prevalence for non-smokers versus $25.1 \%$ for smokers who smoked more than three packs per day [15]. While the data from Deyo and Bass are nearly four decades old and complex samples analysis was not used to address intended variations in sampling the population, they were the first US data to show a potential biological gradient between smoking and back pain. Data from the current study, which used complex samples analysis, support this trend and showed that the prevalence of back pain among Americans was higher with the prevalence of back pain rising from $23.5 \%$ for never smokers to $33.1 \%$ in former smokers and $36.9 \%$ in current smokers. The current study also supports the hypothesis that heavier daily smokers had a higher prevalence of back pain than lighter daily smokers. However, in current some-day smokers, there was no difference in the number of cigarettes smoked per day of those with back pain and those without back pain, which challenges the hypothesis or suggests some other variables are more influential in some-day smokers.

A later US study of data from the 2002 NHIS showed that US adults with low back pain or neck pain had riskier health behaviors than adults without back or neck pain and that the odds of back pain were higher in smokers $(\mathrm{OR}=1.3)$; however, this study did not assess whether there may be a dose-response relationship between smoking levels and back pain [14]. Our data supports and provides an update of NHIS data and provides new information pertaining to the increased prevalence of back pain in NHIS respondents who are daily smokers that smoke more cigarettes.

There are plausible considerations that smoking may have a dose-response relationship with back pain. Biological mechanisms have been hypothesized for the relationship between smoking and back pain and range from neurological to biomechanical mechanisms. It has been suggested that nicotine, through its excitatory effects, alters the perception and threshold for pain, increasing the self-reporting of pain [6]. Thus, greater exposure to nicotine may increase the amount of pain perception. Smoking also increases the level of circulating pro-inflammatory cytokines, which signal the central nervous system and may lead to amplification of pain [7]. It has also been suggested that smoking increases inflammation [24]. Frymoyer, et al. [25] were the first to propose that people with back pain had a greater prevalence of coughing and that there was a "... possibility that mechanical stresses induced by coughing may be relevant to the lowback complaint.” However, this mechanism was not fully supported by Deyo and Bass who used multivariate analysis to control for chronic cough symptoms and found that this did not eliminate the association of smoking with back pain [15]. Other plausible biological mechanisms have been proposed as an underlying basis of an association between smoking and back pain. While this is not an exhaustive review of all possible explanations for this relationship, it does show biological plausibility, one of the criteria necessary when discussing possible doseresponse relationship and epidemiological studies of association [26].

\section{Limitations}

This exploratory study only investigated smoking and low back pain; other biopsychosocial factors that may relate to the amount of smoking or low back pain were not explored. It is possible that some of these variables influenced the prevalence of back pain. However, considering that covariables were likely distributed across this large and complex sample design, we feel that the findings of this study suggest the presence of a biological gradient or doseresponse relationship between smoking and back pain. This study is also limited by the survey data collection and the data are based on self-report and may be influenced by recall bias. One of the inherent limitations to the study is the vague definition of the survey relating to the term "back pain", which could have influenced the prevalence of back pain in the sample. This is a cross-sectional research design and, therefore, cannot imply causation of back pain from smoking [27]. The survey was limited to non-institutionalized and non-military adults. Considering the psychological and physical demands upon these populations, it is possible that 
the estimates provided from this research underreport the prevalence of back pain and the magnitudes of association of some of the independent variables with back pain. This study only investigated adults and, therefore, did not analyze smoking in children and teens. Thus, the results of the present study may not be applicable to other populations.

\section{Generalizability}

The present study provides robust data on a large and representative sample of US adults. There is a higher prevalence of back pain among smokers than former smokers and never smokers and a higher prevalence of back pain among those who smoke more cigarettes. Given the large sample size obtained from independent sampling using a complex sampling strategy and weighting to population estimates using SPSS Complex Samples Analysis, it is felt that the results of this study are generalizable to adults living in the US.

\section{Conclusions}

Further attention is needed to clarify smoking as a potential risk factor associated with the substantial financial and social burden of back pain. The present study found that current smokers who smoked more cigarettes per day had a higher prevalence of back pain. These findings suggest that there might be a biological gradient associated with exposure to smoking cigarettes and back pain in US adults. The risk factor of smoking should be considered as one of many factors when developing research studies or intervention strategies for those with back pain.

\section{Additional Information \\ Disclosures}

Human subjects: Consent was obtained by all participants in this study. Walden University issued approval 11-04-14-0058716. Participants provided consent to participate to the interviewer for the original data collection conducted by the NHIS. This data is released to the public for future secondary analyses and requires no further participant consent. Animal subjects: All authors have confirmed that this study did not involve animal subjects or tissue. Conflicts of interest: In compliance with the ICMJE uniform disclosure form, all authors declare the following: Payment/services info: All authors have declared that no financial support was received from any organization for the submitted work. Financial relationships: All authors have declared that they have no financial relationships at present or within the previous three years with any organizations that might have an interest in the submitted work. Other relationships: All authors have declared that there are no other relationships or activities that could appear to have influenced the submitted work.

\section{Acknowledgements}

The authors are employed by their institutions. Otherwise, there are no potential conflicts of interest to disclose. The views expressed in this article are those of the authors and do not reflect the official policy or position of the Department of Veterans Affairs or the United States Government.

\section{References}

1. Global Burden of Disease Study 2013 Collaborators: Global, regional, and national incidence, prevalence, and years lived with disability for 301 acute and chronic diseases and injuries in 188 countries, 1990-2013: a systematic analysis for the Global Burden of Disease Study 2013. Lancet. 2015, 386:743-800. 10.1016/S0140-6736(15)60692-4

2. Johannes CB, Le TK, Zhou X, Johnston JA, Dworkin RH: The prevalence of chronic pain in United States adults: results of an Internet-based survey. J Pain. 2010, 11:1230-39. 
10.1016/j.jpain.2010.07.002

3. Buchbinder R, Blyth FM, March LM, Brooks P, Woolf AD, Hoy DG: Placing the global burden of low back pain in context. Best Pract Res Clin Rheumatol. 2013, 27:575-89.

10.1016/j.berh.2013.10.007

4. Simon LS: Relieving pain in America: A blueprint for transforming prevention, care, education, and research. J Pain Palliat Care Pharmacother. 2012, 26:197-98.

10.3109/15360288.2012.678473

5. Ackerman WE 3rd, Ahmad M: Effect of cigarette smoking on serum hydrocodone levels in chronic pain patients. J Ark Med Soc. 2007, 104:19-21.

6. Brage S, Bjerkedal T: Musculoskeletal pain and smoking in Norway. J Epidemiol Community Health. 1996, 50:166-69. 10.1136/jech.50.2.166

7. Shiri R, Karppinen J, Leino-Arjas P, Solovieva S, Viikari-Juntura E: The association between smoking and low back pain: a meta-analysis. Am J Med. 2010, 123:87.e7-35.

10.1016/j.amjmed.2009.05.028

8. Andersson H, Ejlertsson G, Leden I: Widespread musculoskeletal chronic pain associated with smoking. An epidemiological study in a general rural population. Scand J Rehabil Med. 1998, 30:185-91.

9. Green BN, Johnson CD: Establishing a theoretical basis for research in musculoskeletal epidemiology: a proposal for the use of biopsychosocial theory in investigations of back pain and smoking. J Chiropr Humanit. 2013, 20:1-8. 10.1016/j.echu.2013.10.004

10. Wai EK, Rodriguez S, Dagenais S, Hall H: Evidence-informed management of chronic low back pain with physical activity, smoking cessation, and weight loss. Spine J. 2008, 8:195-202. 10.1016/j.spinee.2007.10.024

11. Biering-Sørensen F, Thomsen CE, Hilden J: Risk indicators for low back trouble. Scand J Rehabil Med. 1989, 21:151-57.

12. An HS, Silveri CP, Simpson JM, File P, Simmons C, Simeone FA, Balderston RA: Comparison of smoking habits between patients with surgically confirmed herniated lumbar and cervical disc disease and controls. J Spinal Disord. 1994, 7:369-73.

13. Leboeuf-Yde C: Does smoking cause low back pain? A review of the epidemiologic literature for causality. J Manipulative Physiol Ther. 1995, 18:237-43.

14. Strine TW, Hootman JM: US national prevalence and correlates of low back and neck pain among adults. Arthritis Rheum. 2007, 57:656-65. 10.1002/art.22684

15. Deyo RA, Bass JE: Lifestyle and low-back pain. The influence of smoking and obesity . Spine (Phila Pa 1976). 1989, 14:501-506.

16. von Elm E, Altman DG, Egger M, Pocock SJ, Gøtzsche PC, Vandenbroucke JP; STROBE Initiative: The Strengthening the Reporting of Observational Studies in Epidemiology (STROBE) statement: guidelines for reporting observational studies. J Clin Epidemiol. 2008, 61:344-49. 10.1016/j.jclinepi.2007.11.008

17. 2012 National Health Interview Survey (NHIS) Public Use Data Release: NHIS Survey Description. (2013). Accessed: April 5, 2014:

http://ftp.cdc.gov/pub/Health_Statistics/NCHS/Dataset_Documentation/NHIS/2012/srvydesc.pdf.

18. Population estimates: Vintage 2012: National Tables. (2014). Accessed: April 5, 2014: http://www.census.gov/popest/data/historical/2010s/vintage_2012/national.html.

19. Data File Documentation, National Health Interview Survey, 2012 (machine readable data file and documentation). (2013). Accessed: September 21, 2016: http://www.cdc.gov/nchs/nhis/nhis_2012_data_release.htm.

20. Hoy D, Brooks P, Woolf A, Blyth F, March L, Bain C, Baker P, Smith E, Buchbinder R: Assessing risk of bias in prevalence studies: modification of an existing tool and evidence of interrater agreement. J Clin Epidemiol. 2012, 65:934-39. 10.1016/j.jclinepi.2011.11.014

21. Faul F, Erdfelder E, Lang AG, Buchner A: G*Power 3: a flexible statistical power analysis program for the social, behavioral, and biomedical sciences. Behav Res Methods. 2007, 39:17591. 10.3758/BF03193146

22. Cohen JM: Statistical Power Analysis for the Behavioral Sciences, 2nd ed. Cohen JM (ed): L Erlbaum Associates, Hillsdale; 1988.

23. Faul F, Erdfelder E, Buchner A, Lang AG: Statistical power analyses using G*Power 3.1: tests for correlation and regression analyses. Behav Res Methods. 2009, 41:1149-60. 10.3758/BRM.41.4.1149

24. Chung HY, Machado P, van der Heijde D, D'Agostino MA, Dougados M: Smokers in early axial 


\section{Cureus}

spondyloarthritis have earlier disease onset, more disease activity, inflammation and damage, and poorer function and health-related quality of life: results from the DESIR cohort. Ann Rheum Dis. 2012, 71:809-16. 10.1136/annrheumdis-2011-200180

25. Frymoyer JW, Pope MH, Costanza MC, Rosen JC, Goggin JE, Wilder DG: Epidemiologic studies of low-back pain. Spine (Phila Pa 1976). 1980, 5:419-23.

26. Hill AB: The environment and disease: Association or causation?. Proc R Soc Med. 1965, 58:295-300.

27. Understanding the Fundamentals of Epidemiology - An Evolving Text . (2000). Accessed: December 23, 2012: http://www.epidemiolog.net/evolving/TableOfContents.htm. 